\title{
High levels of multiresistance in quinolone resistant urinary tract isolates of Escherichia coli from Norway; a non clonal phenomen?
}

\author{
Linda Strand ${ }^{1,3,4^{*}}$, Andrew Jenkins ${ }^{1,2}$, Ingrid Høgli Henriksen ${ }^{2}$, Anne Gry Allum ${ }^{1,4}$, Nils Grude ${ }^{1,5}$ and Bjørn Erik Kristiansen ${ }^{1,3}$
}

\begin{abstract}
Background: The problem of emerging ciprofloxacin resistance is compounded by its frequent association with multiresistance, the reason for which is not fully understood. In this study we compare multiresistance, clonal similarities and phylogenetic group in urinary tract isolates of Escherichia coli sensitive and resistant to the quinolone antimicrobials nalidixic acid and ciprofloxacin.
\end{abstract}

Results: Quinolone resistant isolates were more resistant to non-quinolone antibiotics than sensitive isolates, with resistance to ampicillin, mecillinam, sulphonamide, trimethoprim, tetracycline, kanamycin and chloramphenicol significantly increased. Fifty-one percent of quinolone-resistant isolates were multiresistant. Although multiresistance was most prevalent (63\%) in isolates showing high-level ciprofloxacin resistance, it was still highly prevalent (41\%) in nalidixic acid resistant isolates with low-level ciprofloxacin resistance. Multiresistance was more frequent among singleton isolates (61\%) than clonal isolates (40\%) of quinolone resistant Escherichia coli. Ciprofloxacin resistance was associated with certain specific clones, among them the globally distributed clonal Group A. However, there was no significant difference in the overall degree of clonality between quinolone sensitive and resistant isolates. Ciprofloxacin resistance was positively associated with phylogroup D and negatively associated with phylogroup B2. This correlation was not associated with clonal isolates.

Conclusion: This study supports earlier findings of association between ciprofloxacin resistance and resistance to other antibiotics. The prevalence of multiresistance in quinolone-resistant isolates that have not yet developed high-level ciprofloxacin resistance suggest that multiresistance arises early in the development of quinolone resistance. This is consistent with exposure to quinolones causing quinolone resistance by mutations and mobilization of multiresistance elements by induction of the SOS response. The spread of clones seems to be less important than previously reported in regard to emergence of quinolone resistance and multiresistance as both are associated primarily with singleton isolates.

Keywords: Multiresistance, E. coli, Clone, Phylogenetic group, CGA, Ciprofloxacin, Nalidixic acid, PFGE

\section{Background}

Since the broad spectrum fluoroquinolone antibiotics were introduced in clinical practice, fluoroquinolone resistant E. coli (FQREC) strains have been isolated with increasing frequency, with prevalence as high as $25-50 \%$ being reported from some southern European countries. In

\footnotetext{
* Correspondence: linda.strand@sthf.no

${ }^{1}$ Unilabs Telelab, Skien, Norway

${ }^{3}$ Department of Microbiology and Virology, University of Tromsø, Tromsø, Norway

Full list of author information is available at the end of the article
}

Norway the prevalence of quinolone resistance, although still low, has doubled to 9.7\% in the period $2005-2009$ [1].

Quinolone resistance is principally due to mutations in the DNA gyrase gene gyrA. Single mutations result in nalidixic acid resistance and moderately raised ciprofloxacin resistance, while full resistance to ciprofloxacin requires two gyrA mutations. Mutations in parC [2-4], mutations that effect efflux pumps or cell permeability and plasmidborne factors may also contribute to resistance [5-7].

The problem of emerging ciprofloxacin resistance is further compounded by its frequent association with multiresistance to other antibiotics [8-13]. The reason for

\section{Biomed Central}


this association is not understood, but several possibilities may be considered. Ciprofloxacin-resistant isolates have been reported to show a high level of clonality $[8,14]$ and resistance has emerged in the multiresistant uropathogenic clonal group A (CGA) [15-20], which suggests a contribution from the spread of multiresistant clones under selection pressure. The agricultural sector has also been suggested as a source of ciprofloxacin resistance [21], in which case multiresistance might arise at source, driven by the extensive use of antibiotic additives as growth stimulants in animal feed. An association between multiresistance and ciprofloxacin resistance might be explained if ciprofloxacin tends to be chosen when therapy with first-line preparations fails due to multiresistance in the target organism. Lastly, quinolones are mutagenic and induce the SOS response which in turn activates the mobility of transposable elements [22-24], suggesting that chromosomal mutations and/or the spread of mobile resistance factors might be involved.

In this study we investigate multiresistance and clonal similarities in ciprofloxacin-sensitive and ciprofloxacinresistant urinary tract isolates of $E$. coli, as well as the nalidixic-acid resistant isolates that are thought to be their precursors. Our results suggest that multiresistance emerges early in the development of ciprofloxacin resistance and place constraints on several of the possible explanations for association between ciprofloxacin resistance and multiresistance. We also reinvestigate our earlier findings suggesting fluoroquinolone resistance and clonal spread and the emergence of fluoroquinolone resistance in clonal group A [20].

\section{Results \\ Ciprofloxacin MIC distribution}

Figure 1 shows the ciprofloxacin MIC distribution of the isolates. The isolates are clearly separated into three subpopulations with peak MICs of 0.032, 0.5 and $64 \mathrm{mg} / \mathrm{L}$. The first population consists of the nalidixic acid sensitive isolates, while the second two contain the nalidixic acid resistant isolates. We have chosen to divide our material on the basis of the natural divisions between the three subpopulations at $0.064-0.125$ and $2-4 \mathrm{mg} / \mathrm{L}$, rather than the clinical breakpoints, which do not coincide with the natural divisions in this material. In order to avoid confusion with the established sensitive-intermediateresistant terminology, we refer to our three subpopulations as Cipro ${ }^{0.032}$ - nalidixic acid sensitive isolates with ciprofloxacin MIC $\leq 0.064$ and modal MIC $0.032 \mathrm{mg} / \mathrm{L}$ $(\mathrm{N}=43)$; Cipro $^{0.5}$ - nalidixic acid resistant isolates with ciprofloxacin $\mathrm{MIC} \geq 0.125$ and $\leq 2$ and modal MIC $0.5 \mathrm{mg} / \mathrm{L}(\mathrm{N}=75)$ and $\mathrm{Cipro}^{64}$ - nalidixic acid resistant, ciprofloxacin resistant isolates with ciprofloxacin MIC $\geq 4$ and modal MIC $32-64 \mathrm{mg} / \mathrm{L}(\mathrm{N}=75)$. $\mathrm{Cipro}^{0.5}$ and
Cipro $^{64}$ isolates are collectively referred to as nalidixic acid resistant isolates.

\section{Resistance to non-quinolone antibiotics}

Nalidixic acid resistant isolates $\left(\mathrm{Cipro}^{0.5}\right.$ and $\mathrm{Cipro}^{64}$ ) expressed a greater number of non-quinolone antibiotic resistances $($ mean $=3.5)$ than nalidixic acid sensitive $\left(\right.$ Cipro $\left.^{0.032}\right)$ isolates $($ mean $=0.4 ; \mathrm{p}<0.001, t$-test), with resistance to all antibiotics tested except nitrofurantoin significantly increased (z-test, $\mathrm{p}<0.05$ ). There was also a slight, but significant difference in the number of resistances between Cipro ${ }^{0.5}$ (mean $=3.2$ ) and Cipro ${ }^{64}$ isolates $($ mean $=3.8 ; p<0.05)$, with significant increase in resistance to trimethoprim and sulphonamide (z-test, $\mathrm{p}<0.05$ ). Antibiotic resistances of the isolates are shown in Table 1.

Fifty-one percent (77/150) of the nalidixic acid resistant isolates were multiresistant, as defined by resistance to four classes of antibiotics in addition to quinolones; $17 \%(25 / 150)$ of the isolates were resistant to five, $6 \%(9 /$ 150) to six and one to seven other antibiotic classes; this isolate showed intermediate susceptibility to mecillinam and was otherwise fully resistant to all antibiotics tested including ciprofloxacin. Eight isolates were fully susceptible only to nitrofurantoin.

Forty percent $(30 / 75)$ of Cipro ${ }^{0.5}$ were multiresistant and $63 \%(47 / 75)$ of Cipro $^{64}$ were multiresistant as defined by resistance to four classes of antibiotics in addition to quinolones. Fourty-nine percent of isolates conforming to the standard definition of ciprofloxacin resistance (MIC $\geq 2 \mathrm{mg} / \mathrm{L}$ ) were multiresistant.

$18 \%(27 / 150)$ of the nalidixic acid resistant isolates shared a distinctive ampicillin-chloramphenicol-sulphonamidetetracycline-trimethoprim multidrug resistance phenotype; these were approximately equally distributed between Cipro $^{0.5}$ (56\%) and Cipro ${ }^{64}(44 \%)$.

Only one nalidixic acid sensitive isolate was multiresistant.

\section{Clonal distribution and phylogroup}

Pulsed-field gel electrophoresis (PFGE) analysis identified 20 clones containing two to 12 isolates (Table 2, Figure 2). Seventy-eight (43\%) of the typable isolates were clonal and 105 (57\%) were singletons. Ten isolates were non-typable due to DNA degradation; these were excluded from statistical analyses of clonal groups. All ten non-typable isolates were of phylogenetic group D, resistant to nalidixic acid, ciprofloxacin (MIC 32-64 mg/L) and sulphonamide and lactose non-fermenting. Eight of these isolates were also resistant to trimethoprim, and seven were resistant to tetracycline and ampicillin. Five (50\%) showed resistance against four or more antibiotics in addition to quinolones.

The two largest clones, clones 1 and 2, contained 12 isolates each and represented 13\% (24/183) of the material. All clone 1 and 2 isolates were nalidixic acid resistant. Clone 1 included four isolates from a suspected outbreak 


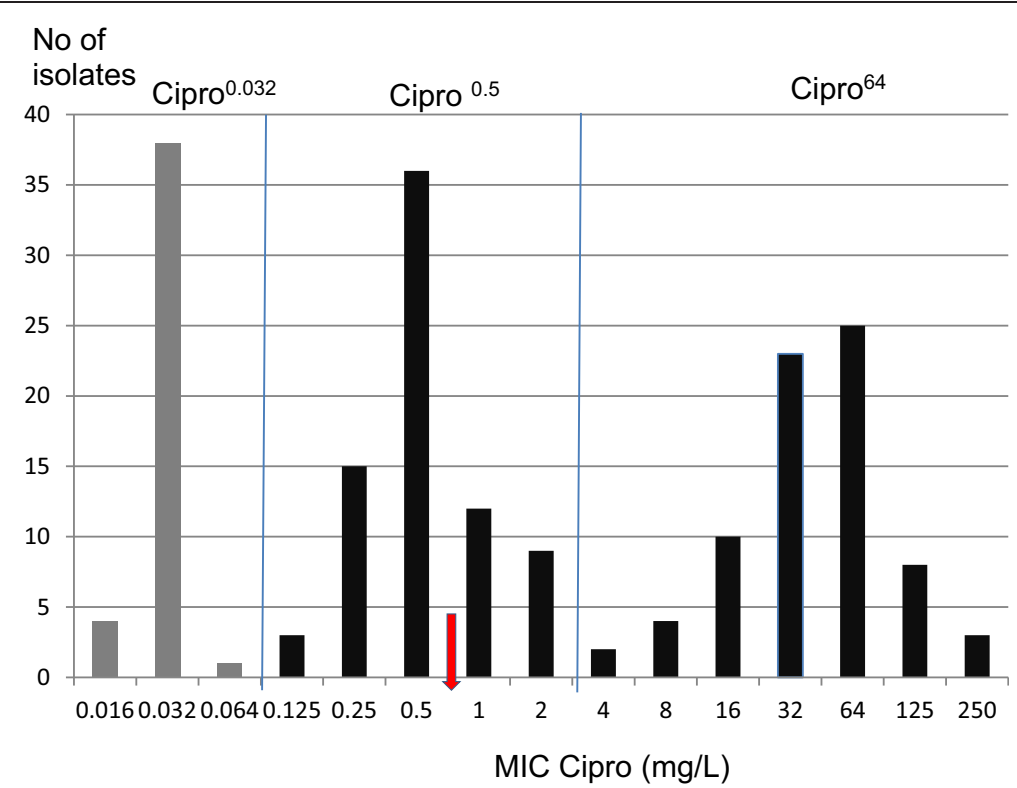

Figure 1 Trimodal distribution of ciprofloxacin MIC. Nalidixic acid sensitive isolates are shown in grey. Vertical lines delineate the subdivisions used for analysis in the rest of the article. The arrow indicates the EUCAST breakpoint between clinical resistance and sensitivity [1].

at an old people's home. Clone 2 was identified as clonal group A. All clones were homogeneous with respect to phylogenetic group.

There was a significant association between clonality and phylogenetic group (Chi-square $=8.850$ with 3 degrees of freedom; $p=0.040$ ) (Figure 3). This can be attributed to the greater prevalence of phylogroup B2 among clonal isolates $\left(+16 \%\left(\mathrm{CI}_{95}: 2-30 \%\right) \mathrm{p}=0.03\right.$, z-test $)$ and of phylogroup A among singleton isolates $\left(+13 \%\left(\mathrm{CI}_{95}: 2-\right.\right.$ $25 \%) \mathrm{p}=0.04$, z-test). The proportion of phylogroup B1 and D was similar among clonal and singleton isolates (Figure 3) and there was no significant difference in clonality between these groups.

Table 1 Antibiotic resistances

\begin{tabular}{cccccccccc}
\hline \multicolumn{1}{c}{ Cipro $^{\mathbf{0 . 0 3 2}}$ ( $\mathbf{n = 4 3 )}$} & Cipro $^{\mathbf{0 . 5}}(\mathbf{n}=\mathbf{7 5})$ & \multicolumn{3}{c}{ Cipro $^{\mathbf{6 4}}(\mathbf{n}=\mathbf{7 5})$} \\
\hline Antibiotic & $\mathrm{S}$ & $\mathrm{I}$ & $\mathrm{R}$ & $\mathrm{S}$ & $\mathrm{I}$ & $\mathrm{R}$ & $\mathrm{S}$ & $\mathrm{I}$ & $\mathrm{R}$ \\
Ampicillin & - & 37 & 6 & - & 22 & 53 & - & 16 & 59 \\
Mecillinam & 41 & - & 2 & 62 & - & 13 & 60 & - & 15 \\
Kanamycin & 42 & - & 1 & 66 & - & 9 & 61 & - & 14 \\
Chloramphenicol & 41 & - & 2 & 50 & - & 25 & 45 & - & 30 \\
Tetracycline & 38 & - & 5 & 27 & 1 & 47 & 19 & 3 & 53 \\
Nitrofurantoin & 43 & - & - & 74 & - & 1 & 74 & - & 1 \\
Sulphonamide & 34 & 3 & 6 & 22 & 1 & 52 & 13 & 0 & 62 \\
Trimethoprim & 38 & - & 5 & 32 & 1 & 42 & 20 & - & 55 \\
\hline
\end{tabular}

Cutoff values between sensitive, intermediate and resistant were: Ampicillin: $\mathrm{I}>0.5, \mathrm{R}>8$; Mecillinam: $\mathrm{R}>8$; Chloramphenicol: $\mathrm{R}>8$; Nitrofurantoin: $\mathrm{R}>64$; Trimethoprim: $\mid>2, R>4$ [25]. Tetracycline: $\mid>4, R>8$; Sulphonamide: $\mid>64$, $\mathrm{R}>128$ [26]. Kanamycin: $\mathrm{R}>10$.
There were no significant differences in the degree of clonality between Cipro ${ }^{0.032}$, Cipro $^{0.5}$ and Cipro ${ }^{64}$ isolates (Chi-square ( 2 d.f. $)=3.966, p=0.138$ ), or between nalidixic acid sensitive and resistant isolates (Chi-square $=0.994$; $\mathrm{P}=0.319$ ), see Figure 4. Ciprofloxacin resistance was, however, associated with specific clones. Two clones contained only Cipro ${ }^{64}$ isolates; six contained only Cipro ${ }^{0.5}$ isolates and three contained only Cipro ${ }^{0.032}$ isolates; five groups contained Cipro ${ }^{0.032}$ and $\mathrm{Cipro}^{0.5}$ isolates and four contained Cipro $^{0.5}$ and Cipro ${ }^{64}$ isolates; no clone contained both Cipro ${ }^{0.032}$ and Cipro ${ }^{64}$ isolates (Table 2).

\section{Clonal group A (CGA)}

All twelve CGA isolates were resistant to nalidixic acid with ciprofloxacin MICs from 0.25 to $8 \mathrm{mg} / \mathrm{L}$, broadly coincident with the Cipro ${ }^{0.5}$ distribution. Four were ciprofloxacin resistant (MIC $\geq 2 \mathrm{mg} / \mathrm{L}$ ). Eight isolates were multiresistant: four were resistant to four, three to five and one to six other antibiotics. This last isolate was fully sensitive only to nitrofurantoin.

Three isolates shared the ampicillin-chloramphenicolsulphonamide-tetracycline-trimethoprim multidrug resistance phenotype predominant in other multiresistant nalidixic acid resistant isolates.

\section{Quinolone resistance and phylogroup}

Ciprofloxacin resistance was positively associated with phylogroup D and negatively associated with phylogroup B2 (Figure 5). Phylogroup D was significantly more prevalent $(44 \%, 33 / 75)$ among Cipro ${ }^{64}$ isolates than among Cipro $^{0.032}$ isolates $(23 \%, 10 / 43)\left(+21 \%, \mathrm{CI}_{95}: 2 \%-38 \%\right.$; 
Table 2 Ciprofloxacin resistance and phylogenetic groups of clones

\begin{tabular}{|c|c|c|c|c|c|c|}
\hline & $\mathrm{N}$ & Cipro $^{0.032}$ & Cipro $^{0.5}$ & Cipro $^{64}$ & Phylogroup & \\
\hline Clone 1 & 12 & - & 1 & 11 & B2 & $\begin{array}{l}\text { Includes } \\
\text { outbreak }\end{array}$ \\
\hline Clone 2 & 12 & - & 10 & 2 & $\mathrm{D}$ & $C G A$ \\
\hline Clone 3 & 7 & - & - & 7 & $\mathrm{D}$ & \\
\hline Clone 4 & 5 & 5 & - & - & B2 & \\
\hline Clone 5 & 6 & - & 6 & - & B2 & \\
\hline Clone 6 & 4 & 1 & 3 & - & D & \\
\hline Clone 7 & 4 & 1 & 3 & - & B2 & \\
\hline Clone 8 & 3 & - & 1 & 2 & A & \\
\hline Clone 9 & 3 & 3 & - & - & B1 & \\
\hline Clone 10 & 2 & - & 2 & - & B2 & \\
\hline Clone 11 & 2 & - & 2 & - & B2 & \\
\hline Clone 12 & 2 & - & - & 2 & B1 & \\
\hline Clone 13 & 2 & 1 & 1 & - & $A$ & \\
\hline Clone 14 & 2 & - & 2 & - & B2 & \\
\hline Clone 15 & 2 & 1 & 1 & - & B2 & \\
\hline Clone 16 & 2 & - & 1 & 1 & $A$ & \\
\hline Clone 17 & 2 & - & 2 & - & $A$ & \\
\hline Clone 18 & 2 & 2 & - & - & $\mathrm{D}$ & \\
\hline Clone 19 & 2 & 1 & 1 & - & B2 & \\
\hline Clone 20 & 2 & - & 2 & - & D & \\
\hline
\end{tabular}

z-test, $\mathrm{p}=0.04$ ) while the prevalence of phylogroup B2 was lower $(21 \%, 16 / 75)$ in Cipro $^{64}$ than in Cipro ${ }^{0.032}$ (56\%, 24/43) (-35\%, $\mathrm{CI}_{95}: 17 \%-53 \%$; z-test, $\left.\mathrm{p}<0.001\right)$.

In order to investigate whether this is due to the effect of clones, we compared the correlation between phylogroup and quinolone resistance in singleton and clonal isolates. Phylogroup was correlated with quinolone resistance in singleton isolates (chi-squared, 6 d.f. $=17.009, \mathrm{p}=0.009$ ), but not in clonal isolates (Chi-square, 6 d.f. $=8.626$; $\mathrm{p}=0.196)$. Phylogroup B2 was $45 \%\left(\mathrm{CI}_{95}: 23 \%-67 \%\right)$ less prevalent in $\mathrm{Cipro}^{64}$ than in Cipro ${ }^{0.032}$ singleton isolates ( $p<0.001$, $z$-test); the prevalence of phylogroups $\mathrm{A}, \mathrm{B} 1$ and $\mathrm{D}$ was increased but did not achieve statistical significance ( $\mathrm{p}>0.2$, z-test).

\section{Multiresistance in clonal and singleton isolates}

In quinolone-resistant isolates, multiresistance, as defined by resistance to four classes of non-quinolone antibiotics, was found in 25 of 63 (40\%) clonal isolates and 47/77 (61\%) singleton isolates. This is statistically significant $(\mathrm{p}=0.019)$. Non-typable isolates were not included.

\section{Discussion}

This study supports earlier findings [8-13] of an association between ciprofloxacin resistance and resistance to other antibiotics, frequently in the form of multiresistance. Association was found with resistance to all antibiotics tested except nitrofurantoin. This, however, applies not only to ciprofloxacin-resistant isolates but also to nalidixic acid resistant isolates with low ciprofloxacin resistance levels, which may be presumed to be isolates with only one gyrA mutation. This indicates that multiresistance arises at an early stage in the stepwise development of ciprofloxacin resistance. As far as we are aware, association between nalidixic acid resistance and multiresistance has not previously been reported.

Eight quinolone-resistant isolates were susceptible only to nitrofurantoin; one isolate showed only intermediate susceptibility to mecillinam and was otherwise fully resistant to all antibiotics tested. These isolates were not clonally related. However, one isolate belonged to clonal group A and resembled two highly resistant CGA isolates previously isolated by us in 2003 [20].

The cause of the association between multiresistance and quinolone resistance is not known. However, a model based on spread of multiresistant clones is not consistent with our data. If the emergence of multiresistant quinolone-resistant isolates were due to clonal spread, we would expect multiresistance to be more prevalent among clonal isolates. This is the reverse of what we observe in this study. Singleton isolates are significantly more multiresistant than clonal isolates.

Neither do our results confirm previous findings of increased clonality among ciprofloxacin-resistant isolates $[8,14]$. Ciprofloxacin resistant isolates were no more clonal than sensitive isolates. Ciprofloxacin resistant clones did however occur, suggesting that clonal spread does make some contribution to the spread of ciprofloxacin resistance. Cagnacci et al. assigned 34\% of 148 ciprofloxacin resistant isolates to two clonal groups using a combination of methods including multilocus sequence typing (MLST) [14]. This is a higher degree of clonality than we observe, despite their collection being both more geographically dispersed and collected over a longer time period. However their study includes material from the Mediterranean region where quinolone resistance is well established, while our material is from a low-prevalence region where emergence of quinolone resistance may still be dominant over spread.

Clonal group A was the second most prevalent clone found in our material, representing 6\% (12/193) of the isolates. All CGA isolates were quinolone resistant and one third were ciprofloxacin resistant, confirming the emergence of fluoroquinolone resistance in CGA that we [20] and others [11] have previously observed. Although CGA is considered a multiresistant clone, CGA isolates in this material were not significantly more multiresistant than other quinolone resistant isolates ( $p>0.5$, z-test). In 2001, CGA comprised 19\% of quinolone sensitive isolates, while in this material, from 2005, quinolone-sensitive 
a

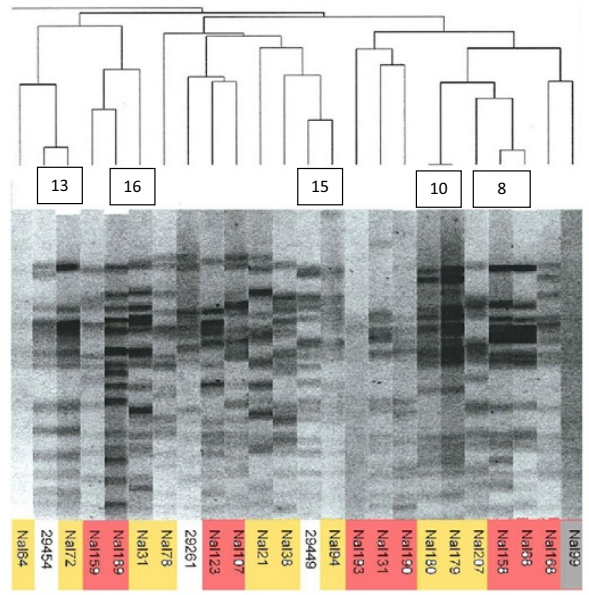

b
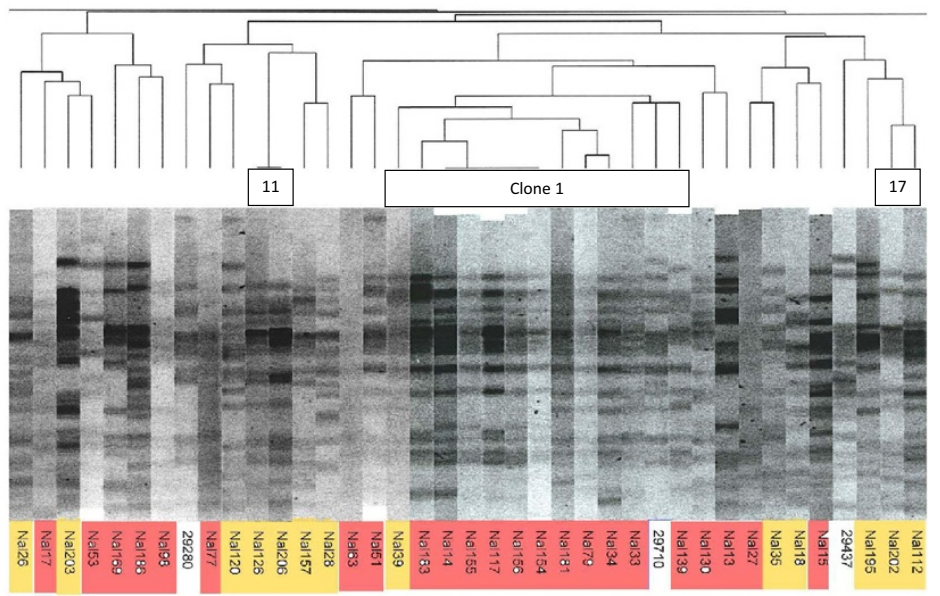

e
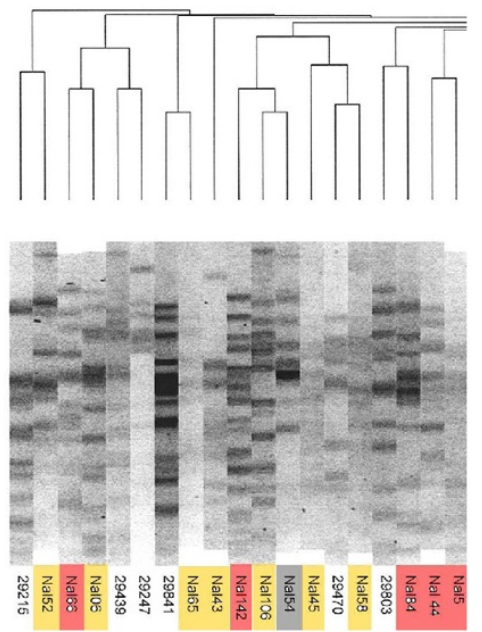

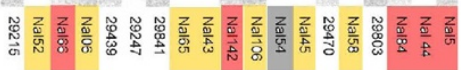

d
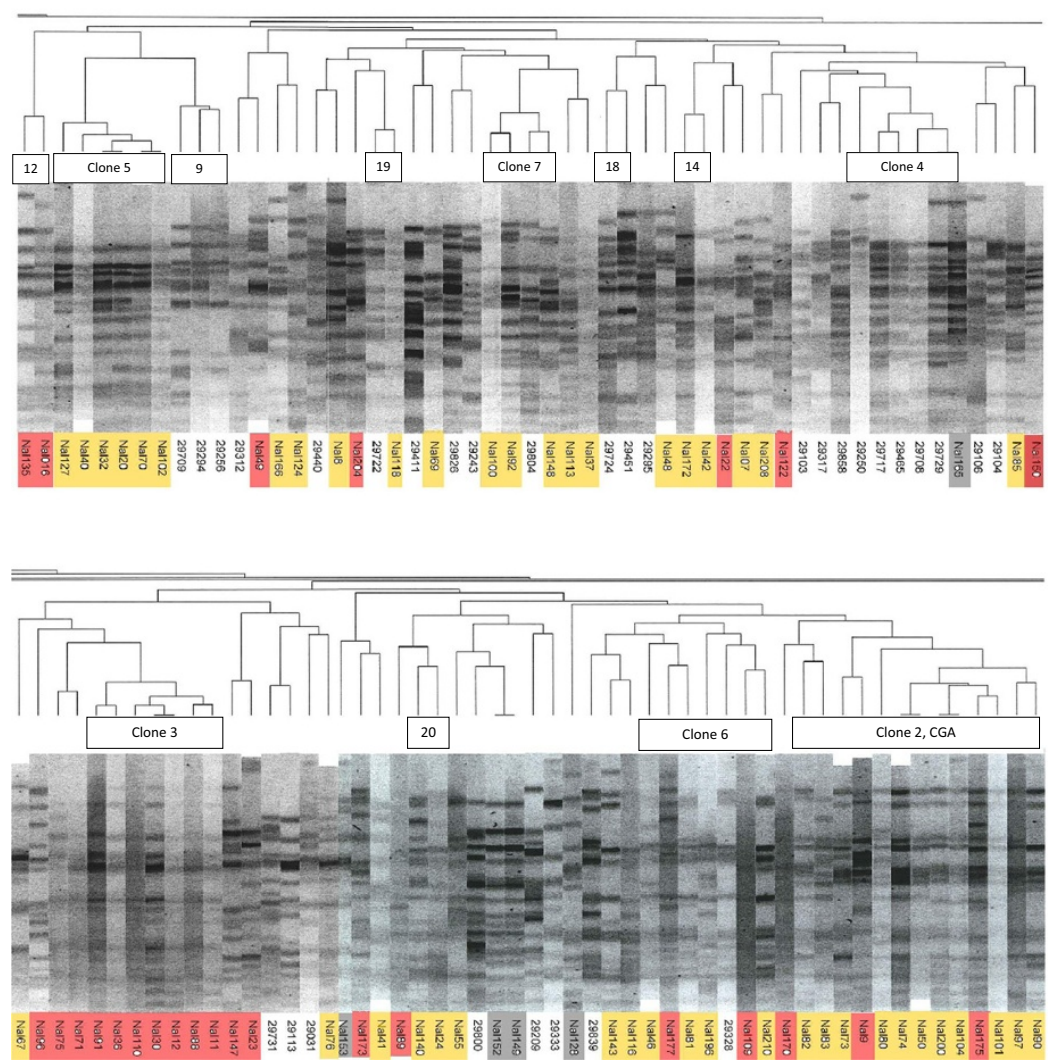

Figure 2 Xbal PFGE patterns of the isolates. (a-e) Highlighting of the isolate code indicates cipro ${ }^{64}$ (red), cipro 0.5 (yellow) and cipro ${ }^{0.032}$ (not highlighted). Grey indicates that the sample is excluded from the study. Panels a - e are parts of a single image which has been divided for convenience of presentation. The left border of panel $a$ is the right border of panel b, and so forth. Clone numbers are placed between the dendrogram and the gel pictures. In a few cases, patterns grouped with a clone in the dendrogram do not satisfy our criteria for clone inclusion ( $<7$ bands difference from the prototype). Therefore the number of patterns marked may differ from the number given in Table 2.

CGA was not found. This difference is statistically significant $(\mathrm{p}=0.011$, $\mathrm{z}$-test $)$ and strongly suggests that a shift to quinolone resistance in CGA has occurred.

The four largest clones among the quinolone resistant isolates, clones 1, 2 (CGA, discussed above), 3 and 5 belonged to the uropathogenic phylogenetic groups B2 and $\mathrm{D}$ and comprised $25 \%$ of the quinolone-resistant isolates. The twelve clone 1 isolates belonged to phylogenetic group B2. Four of these isolates were epidemiologically associated. Clone 3 (7 isolates) belonged to phylogroup 


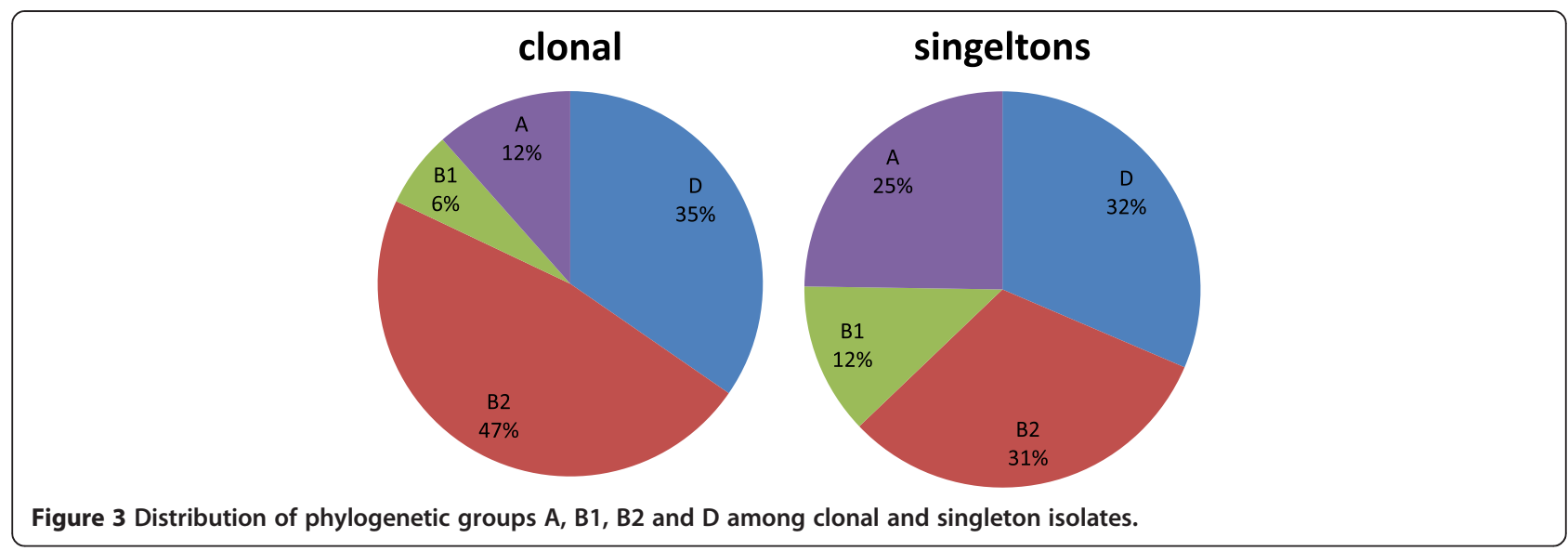

D. Clone 5 (6 isolates) belonged to phylogenetic group B2. Clones 1, 2 (CGA) and 3 were also present among fluoroquinolone resistant isolates from 2003 [8]. The clones did not display uniform resistance patterns, although all of clone 3 and $66 \%$ of clone 2 (CGA) isolates were trimethoprim-sulfonamide resistant.

Quinolone resistance was positively correlated with phylogenetic group D and negatively correlated with phylogenetic group B2 as has previously been reported [27-29]. This correlation was confined to non-clonal isolates, which implies that phylogroup D more readily acquires quinolone resistance mutations than phylogroup B2 and that the spread of clones is of lesser importance, in contrast to what has previously been suggested $[8,14]$.

The predominant multiresistance phenotype, resistance to ampicillin, sulphonamide, trimethoprim, tetracycline and chloramphenicol was found in 19\% of quinolone resistant isolates. It was widely disseminated among clonally unrelated isolates. This resembles the resistance pattern described in outbreak strains of O15:K52:H1 and CGA $[16,17]$ and was found in three of the CGA isolates

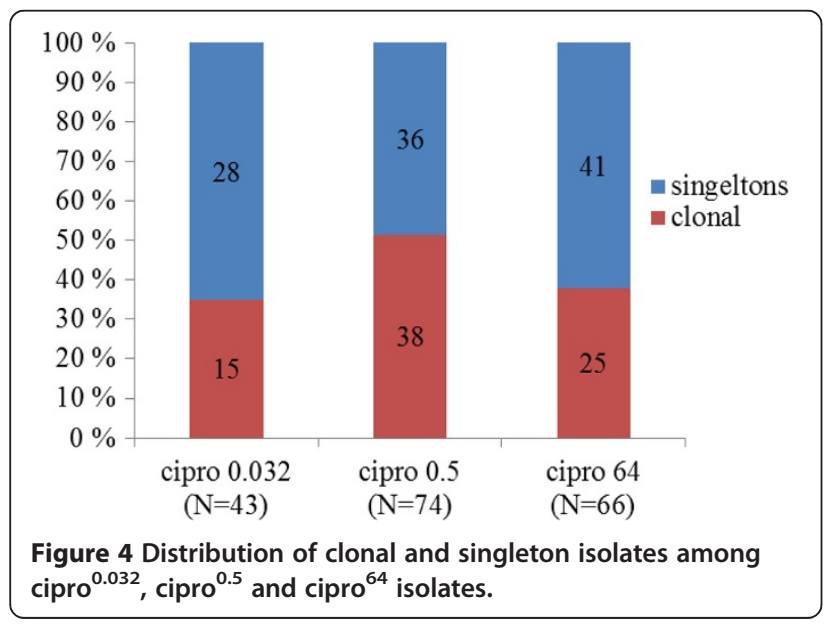

in this study. The genes giving this resistance phenotype have been located in a chromosomal integration hot spot in CGA by Lescat and coworkers [30].

This multiresistance pattern is one typically associated with transposable elements and plasmids, suggesting an association between quinolone resistance and mobile genetic elements and lending support to the idea that multiresistance arises by the mobilization of transposable elements caused by induction of the SOS response during quinolone therapy, an effect that has been suggested by several authors [22-24]. In this model, it is exposure to quinolones that causes mobilization of multiresistance elements, which is consistent with an early emergence of multiresistance, as observed in this study.

Fluoroquinolones are popular antibiotics; easily administered, cheap, broadly applicable, quick acting and showing low toxicity. However, in view of the emergence of resistance in pathogenic clonal groups such as CGA and the tendency of fluoroquinolones to provoke multiresistance, some caution in their use and monitoring of their effects is indicated.

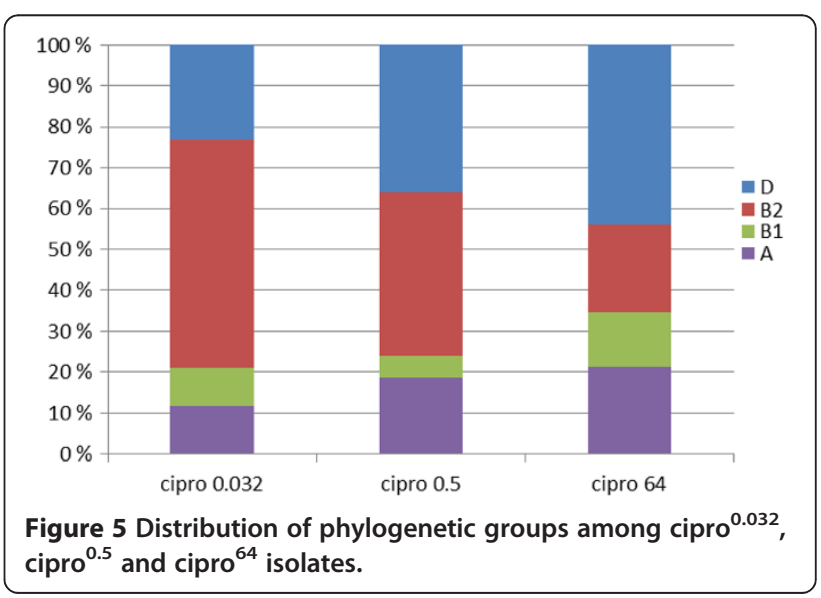




\section{Conclusions}

This study supports earlier findings of association between ciprofloxacin resistance and resistance to other antibiotics. We found that emergence of multiresistance arises early in the development of quinolone resistance and is mostly associated with singleton isolates. This is consistent with exposure to quinolones causing quinolone resistance by mutations and mobilization of multiresistance elements by induction of the SOS response. The spread of clones seems to be less important than previously reported in regard to emergence of quinolone resistance and multiresistance.

\section{Methods}

\section{E. coli isolates}

E. coli isolates were collected from community urine samples submitted to Telelab, a microbiology laboratory serving hospitals and primary practices in the counties of Telemark and Vestfold, south-eastern Norway for routine bacteriological analysis and showing significant bacteruria (pure culture of $\geq 10000$ bacteria/ml). The material consisted of 150 consecutive nalidixic acid resistant isolates and 43 randomly-chosen nalidixic acid sensitive isolates collected in the same period in 2005. E. coli was identified by colony morphology and biochemical profile using the three tube test [31].

\section{Clonal analysis}

Pulsed-field gel electrophoresis using restriction endonuclease $\mathrm{XbaI}$ was performed as previously described $[8,32]$. Gels were photographed, scanned as TIFF files, and imported into Gelcompar software (Applied Maths, Sint-Martin-Latem, Belgium). A dendrogram was constructed using band-based comparisons with Dice coefficient and cluster analysis with the unweighted pair-group method with arithmetic average (UPGMA); $1 \%$ position tolerance and 3\% optimization settings were used. PFGE patterns differing by less than seven bands from prototype members were assigned to the same clone.

\section{Phylogrouping}

Phylogrouping by triplex-PCR was performed as previously described [8,32].

\section{Resistance}

MICs of ampicillin, mecillinam, nitrofurantoin, sulphonamide, trimethoprim, tetracycline, nalidixic acid, ciprofloxacin, kanamycin and chloramphenicol were measured by agar dilution on iso-sensitest medium (Oxoid, Basingstoke, UK). MIC was defined as the lowest concentration that completely inhibited growth.

\section{Clonal group A}

Isolates were identified by detection of the fumC SNP C288T by PCR as previously described $[18,20]$.

\section{Statistical analyses}

The Pearson Chi-square test was used to detect significant differences in clonality and phylogenetic distribution between nalidixic acid resistant and sensitive isolates. The $t$-test was used to test for significant difference in multiplicity of antibiotic resistances. The z-test was used to compare proportions.

\section{Competing interests}

The authors report no competing interest.

\section{Authors' contributions}

Phylogrouping was done by $\mathrm{HH}$ and AGA. PFGE was done by $\mathrm{HH}$. CGA testing was done by $A G A$ and $I H H$. Antibiotic resistance testing was conducted by $\mathrm{HH}$. The study was planned by BEK, NG, AJ and LS. Data analysis, statistical analysis and drafting of the manuscript were done by LS with the assistance of AJ. NG and BEK provided critical comments on the manuscript. All authors read and approved the final manuscript.

\section{Acknowledgements}

This work was supported by grants from the Scandinavian Society for Antimicrobial Chemotherapy and Norsk Overvåkingssystem for Antibiotikaresistente Mikrober (NORM), tax relief via the Skattefunn system and Telelab's internal funds.

\section{Author details}

${ }^{1}$ Unilabs Telelab, Skien, Norway. ${ }^{2}$ Department of Environmental and Health Sciences, Telemark University College, $B \varnothing$, Norway. ${ }^{3}$ Department of Microbiology and Virology, University of Troms $\varnothing$, Troms $\varnothing$, Norway. ${ }^{4}$ Present address: Telemark Hospital, Medical and Environmental Genetics Unit, Skien, Norway. ${ }^{5}$ Present address: Department of Medical Microbiology, Vestfold Trust Hospital, Tønsberg, Norway.

Received: 26 January 2014 Accepted: 28 May 2014

Published: 19 June 2014

\section{References}

1. Antimicrobial resistance interactive database, EARS-Net. [http://www. ecdc.europa.eu/en/healthtopics/antimicrobial_resistance/database/Pages/ database.aspx]

2. Heisig P: Genetic evidence for a role of parC mutations in development of high-level fluoroquinolone resistance in Escherichia coli. Antimicrob Agents Chemother 1996, 40:879-885.

3. Hopkins KL, Davies RH, Threlfall EJ: Mechanisms of quinolone resistance in Escherichia coli and Salmonella: recent developments. Int I Antimicrob Agents 2005, 25:358-373.

4. Jacoby GA: Mechanisms of resistance to quinolones. Clin Infect Dis 2005, 41(Suppl 2):120-126.

5. Perichon B, Courvalin P, Galimand M: Transferable resistance to aminoglycosides by methylation of G1405 in 16S rRNA and to hydrophilic fluoroquinolones by QepA-mediated efflux in Escherichia coli. Antimicrob Agents Chemother 2007, 51:2464-2469.

6. Piddock LJ: Clinically relevant chromosomally encoded multidrug resistance efflux pumps in bacteria. Clin Microbiol Rev 2006, 19:382-402.

7. Robicsek A, Jacoby GA, Hooper DC: The worldwide emergence of plasmid-mediated quinolone resistance. Lancet Infect Dis 2006, 6:629-640.

8. Grude N, Strand L, Mykland H, Nowrouzian FL, Nyhus J, Jenkins A, Kristiansen B-E: Fluoroquinolone-resistant uropathogenic Escherichia coli in Norway: evidence of clonal spread. Clin Microbiol Infect 2008, 14:498-500.

9. Karlowsky JA, Hoban DJ, Decorby MR, Laing NM, Zhanel GG: Fluoroquinolone-resistant urinary isolates of Escherichia coli from outpatients are frequently multidrug resistant: results from the North 
American urinary tract infection collaborative alliance-quinolone resistance study. Antimicrob Agents Chemother 2006, 50:2251-2254.

10. Ruiz del Castillo B, Vinué L, Román EJ, Guerra B, Carattoli A, Torres C, MartinezMartinez L: Molecular characterization of multiresistant Escherichia coli producing or not extended-spectrum $\beta$-lactamases. BMC Microbiol 2013, 13:84.

11. Johnson JR, Menard M, Johnston B, Kuskowski MA, Nichol K, ZhanelL GG: Epidemic clonal groups of Escherichia coli as a cause of antimicrobialresistant urinary tract infections in Canada, 2002 to 2004. Antimicrob Agents Chemother 2009, 53:2733-2739.

12. Platell JL, Johnson JR, Cobbold RN, Trott DJ: Multidrug-resistant extraintestinal pathogenic Escherichia coli of sequence type ST131 in animals and food. Vetmic 2011, 153:99-108,

13. Giufre M, Graziani C, Accogli M, Luzzi I, Busani L, Cerquetti M, on behalf of the Escherichia coli Study Group: Escherichia coli of human and avian origi: detection of clonal groups associated with fluoroquinolone and multidrug resistance in Italy. J Antimicrob Chemother 2012, 67:860-867.

14. Cagnacci S, Gualco L, Debbia E, Schito GC, Marchese A: European emergence of ciprofloxacin-resistant Escherichia coli clonal groups O25: H4-ST 131 and 015:K52:H1 causing community-acquired uncomplicated cystitis. J Clin Microbiol 2008, 46:2605-2612.

15. Manges AR, Johnson JR, Foxman B, O'Bryan TT, Fullerton KE, Riley LW: Widespread distribution of urinary tract infections caused by a multidrugresistant Escherichia coli clonal group. N Engl J Med 2001, 345:1007-1013.

16. Manges AR, Dietrich PS, Riley LW: Multidrug-resistant Escherichia coli clonal groups causing community-acquired pyelonephritis. Clin Infect Dis 2004, 38:329-334.

17. Johnson JR, Manges AR, O'Bryan TT, Riley LW: A disseminated multidrugresistant clonal group of uropathogenic Escherichia coli in pyelonephritis. Lancet 2002, 359:2249-2251.

18. Johnson JR, Owens K, Manges AR, Riley LW: Rapid and specific detection of Escherichia coli clonal group A by gene-specific PCR. J Clin Microbiol 2004, 42:2618-2622.

19. Johnson JR, Murray AC, Kuskowski MA, Schubert S, Prére M-F, Picard B, Colodner R, Raz R, and the Trans-Global Initiative for Antimicrobial Resistance Analysis (TIARA) Investigators: Distribution and characteristics of Escherichia coli clonal group A. Emerg Infect Dis 2005, 11:141-145.

20. Strand L, Jenkins A, Grude N, Allum A-G, Mykland H-C, Nowrouzian FL, Kristiansen B-E: Emergence of fluoroquinolone-resistant clonal group A: clonal analysis of Norwegian and Russian E. coli isolates. APMIS 2010, 118:571-577.

21. Johnson JR, Kuskowski MA, Menard M, Gajewski A, Xercavins M, Garau J: Similarity between human and chicken Escherichia coli isolates in relation to ciprofloxacin resistance status. J Infect Dis 2006, 194:71-78.

22. Walsh TR: Combinatorial genetic evolution of multiresistance. Curr Opin Microbiol 2006, 9:476-482.

23. Guerin E, Cambray G, Sanchez-Alberola N, Campoy S, Erill I, Da Re S, Gonzalez-Zorn B, Barbé J, Ploy M-C, Mazel D: The SOS response controls integron recombination. Science 2009, 324:1034.

24. Beaber JW, Hochhut B, Waldor MK: SOS response promotes horizontal dissemination of antibiotic resistance genes. Nature 2004, 427:72-74.

25. AFA: http://www.unn.no/afa/category $10274 . h t m l$ (2009).

26. AFA: http://www.unn.no/afa/category10274.html (2007).

27. Johnson JR, Van Der Schee C, Kuskowski MA, Goessens W, Van Belkum A: Phylogenetic background and virulence profiles of fluoroquinoloneresistant clinical Escherichia coli isolates from the Netherlands. J Infect Dis 2002, 186:1852-1856.

28. Johnson JR, Kuskowski MA, O'Bryan TT, Colodner R, Raz R: Virulence genotype and phylogenetic origin in relation to antibiotic resistance profile among Escherichia coli urine sample isolates from Israeli women with acute uncomplicated cystitis. Antimicrob Agents Chemother 2005, 49:26-31.

29. Moreno E, Prats G, Sabate M, Perez T, Johnson JR, Andreu A: Quinolone, fluoroquinolone and trimethoprim/sulfamethoxazole resistance in relation to virulence determinants and phylogenetic background among uropathogenic Escherichia coli. J Antimicrob Chemother 2006, 57:204-211.

30. Lescat M, Calteau A, Hoede C, Barbe V, Touchon M, Rocha E, Tenaillon O, Médigue C, Johnson JR, Denamur E: A module located at a chromosomal integration hot spot is responsible for the multidrug resistance of a reference strain from Escherichia coli clonal group A. Antimicrob Agents Chemother 2009, 53:2283-2288.
31. Lassen J: Rapid identification of gram-negative rods using a three-tube method combined with a dichotomic key. Acta Pathol Microbiol Scand Supp/ 1975, 83:525-533.

32. Grude N, Potaturkina-Nesterova NI, Jenkins A, Strand L, Nowrouzian FL, Nyhus J, Kristiansen B-E: A comparison of phylogenetic group, virulence factors and antibiotic resistance in Russian and Norwegian isolates of Escherichia coli from urinary tract infection. Clin Microbiol Infect 2007, 13:208-211.

doi:10.1186/1756-0500-7-376

Cite this article as: Strand et al: High levels of multiresistance in quinolone resistant urinary tract isolates of Escherichia coli from Norway; a non clonal phenomen? BMC Research Notes 2014 7:376

\section{Submit your next manuscript to BioMed Central and take full advantage of:}

- Convenient online submission

- Thorough peer review

- No space constraints or color figure charges

- Immediate publication on acceptance

- Inclusion in PubMed, CAS, Scopus and Google Scholar

- Research which is freely available for redistribution

Submit your manuscript at www.biomedcentral.com/submit
C BioMed Central 